\title{
Effect of the bi-chromatic ac magnetic field on the quantum spin chain with an impurity
}

\author{
A.A. Zvyagin $\frac{11,2]}{2}$ \\ 1 Max-Planck-Institut für Physik komplexer Systeme, Noethnitzer Str., 38, D-01187, Dresden, Germany \\ 2 B.I. Verkin Institute for Low Temperature Physics and Engineering of the National Academy of Sciences of \\ Ukraine, Nauky Ave., 47, Kharkiv, 61103, Ukraine
}

Received June 10, 2020, in final form August 12, 2020

\begin{abstract}
The linear response of the quantum spin chain with the impurity to the bi-chromatic ac magnetic field has been calculated. The linearly polarized component of the ac field produces additional resonance absorption with respect to the one caused by the circularly polarized field. The strength of the absorption oscillates with the amplitude and the frequency of the linearly polarized magnetic field. Additional resonance absorption is weaker than the main absorption at the frequency of the circularly polarized field. The absorption due to the impurity can be seen in the absorptions at local levels and the delocalized states.
\end{abstract}

Key words: bi-chromatic ac magnetic field, quantum spin chain, impurity

\section{Introduction}

During the recent years due to the development of spintronics and studies of quantum spin liquids [1,2] the interest to quantum spin systems has grown considerably. Unlike higher-dimensional models, their low-dimensional quantum counterparts permit to obtain exact (non-perturbative) results [3]. The necessity of non-perturbative studies in low-dimensional quantum systems is caused by the enhanced quantum and thermal fluctuations there, due to the features in the density of states [4]. Integrable models are important not only for the pure theory. They are also used in a number of areas, which are described by that theory. Among such systems there are real quasi-low-dimensional spin systems, topological insulators, ultracold atoms in low-dimensional optical traps, sets of qubits (subjects of quantum information and computation) in quantum computers, etc.

It is also known that dynamical properties of an impurity are not only the function of that single ion, but of its environment [5]. That feature was observed, e.g., for atoms closed to plane interfaces, in cavities and photonic crystals, etc. [6-9].

Recently, the response of quantum spin system to the multi-frequency magnetic field directed along the same axis was calculated [10]. In this paper, using the exact quantum solution, the resonance absorption of the bi-chromatic ac magnetic field with different polarizations by a one-dimensional spin system with an impurity is calculated. As the example, the so-called XX spin- $1 / 2$ chain is considered. We show that the absorption manifests contributions from the combined resonances, which appear due to the coherent interference resonance effects of the bi-chromatic field. On the other hand, we show how coupling of the impurity to the spin chain can strongly affect the shape of the resonance absorption spectra, different from the ones of the homogeneous chain.

\section{Interaction of quantum spins with the bi-chromatic ac magnetic field}

Consider now the action of the bi-chromatic magnetic field $\mathbf{H}(t)=-H_{1}[\mathbf{x} \cos (\omega t)+\mathbf{y} \sin (\omega t)]-$ $H_{2} \mathbf{z} \cos (\Omega t)$ i.e., the ac magnetic field has the component with the frequency $\omega$ and the magnitude $H_{1}$, 
circular polarized in the $x y$ plane, and the component with the frequency $\Omega$ and the magnitude $H_{2}$, linear polarized parallel to the $z$-axis. The coupling of the ac magnetic field to the system can be described by the Hamiltonian

$$
\begin{aligned}
\mathcal{H}_{t}= & -\left(g \mu_{\mathrm{B}} H_{1} / 2\right) \sum_{n}\left(S_{n}^{+} \mathrm{e}^{-\mathrm{i} \omega t}+\text { H.c. }\right) \\
& -g^{z} \mu_{\mathrm{B}} H_{2} \cos (\Omega t) \sum_{n} S_{n}^{z},
\end{aligned}
$$

where $\mu_{\mathrm{B}}$ is Bohr's magneton, $g$ and $g^{z}$ are components of the effective $g$-factor of the spin system, $S_{n}^{x, y, z}$ are the operators of the projections of the spin $S$ located at the $n$-th site, and $S_{n}^{ \pm}=S_{n}^{x} \pm \mathrm{i} S_{n}^{y}$. From now on, let us denote $g \mu_{\mathrm{B}} H_{1} \equiv h_{1}$ and $g^{z} \mu_{\mathrm{B}} H_{2} \equiv h_{2}$.

Consider the action of the ac magnetic field on the spin- $1 / 2$ system described by the Hamiltonian

$$
\mathcal{H}_{0}=\sum_{n, j}\left[J_{n} \mathbf{S}_{n} \cdot \mathbf{S}_{n+j}+J_{n}^{z} S_{n}^{z} S_{n+j}^{z}\right]-g^{z} \mu_{\mathrm{B}} H_{d c} \sum_{n} S_{n}^{z},
$$

where $\mathbf{a} \cdot \mathbf{b}$ denotes the scalar product, $J_{n}$ is the constant of the isotropic exchange, $J_{n}^{z}$ is the constant of the inter-ion magnetic anisotropy, and $H_{d c}$ is the value of the dc magnetic field directed along $z$ axis.

Let us use the unitary transformation

$$
U=\exp \left[\mathrm{i} h_{2} \sin (\Omega t) \sum_{n} S_{n}^{z} / \hbar \Omega\right] .
$$

The time-dependent part of the considered Hamiltonian can be transformed to

$$
\mathcal{H}_{t}^{(1)}=-\left(h_{1} / 2\right) \sum_{n}\left\{S_{n}^{+} \exp \left[-\mathrm{i} \omega t+\mathrm{i}\left(h_{2} / \hbar \Omega\right) \sin (\Omega t)\right]+\text { H.c. }\right\} .
$$

Then, we can use the equality

$$
\exp [\mathrm{i} z \sin (\Omega t)]=\sum_{m=-\infty}^{\infty} J_{m}(z) \exp (\mathrm{i} m \Omega t),
$$

where $J_{m}(z)$ is the Bessel function. Notice that $J_{-m}(z)=-J_{m}(z)$. It follows that

$$
\mathcal{H}_{t}^{(1)}=-\left(h_{1} / 2\right) \sum_{n} \sum_{m=-\infty}^{\infty} J_{m}\left(h_{2} / \hbar \Omega\right)\left\{S_{n}^{-} \exp [-\mathrm{i}(\omega-m \Omega) t]+\text { H.c. }\right\} .
$$

Such a transformation can be realized for the homogeneous spin system $J_{n}=J$ and $g_{n}^{z}=g^{z}$. Moreover, it is easy to show that similar (though space-inhomogeneous) transformation can be performed for the system with a single impurity, see below. Then, as usually we can use the transformation to the rotated frame with the frequency $\omega-m \Omega$. The final Hamiltonian has no explicit time dependence. In fact, this expression implies that the effect of the bi-chromatic ac magnetic field is reduced to the effect of the number of circularly polarized fields with the magnitude $h_{1} J_{m}\left(h_{2} / \hbar \Omega\right)$ and the frequencies $\omega-m \Omega$. The linear polarized component of the field produces additional resonances with $m= \pm 1, \pm 2, \ldots$, with respect to the case of the only circular polarized field, which corresponds to the case $m=0$ and $h_{2}=0$. The number of possible resonances is determined by the relative relations between the values $H_{d c}, J_{n} / \hbar$, and $\omega$ and $\Omega$. The absolute value of the Bessel function is the decaying oscillating function of its argument. Hence, the absorption by the spin chain under the action of the bi-chromatic ac magnetic field must show quasi-periodic modulations as a function of the magnitude and the frequency of the linearly polarized field. Contributions from the resonances with larger values of $m$ become essential for larger values of $h_{2} / \hbar \Omega$. 


\section{The spin chain with an impurity}

Now, we restrict ourselves to the one-dimensional case (for simplicity we consider the semi-infinite spin- $1 / 2$ chain) with the interaction $J_{n}$ in $\mathcal{H}_{0}$ limited to the nearest neighboring spins $j=1$. Moreover, let us consider the case with $J_{n}^{z}=-J_{n}$, and for the sites for $n=1, \ldots, J_{n}=J$, and $g_{n}^{z}=g^{z}$. On the other hand, for the site $n=0$ we consider $J_{0}=J^{\prime}$ and $H_{d c}=H_{l}$. It means that we consider the semi-infinite spin-1/2 isotropic XY chain with the impurity located at the edge of the chain. The impurity distinguishes from the other sites by the coupling $J^{\prime} \neq J$ and by the effective local magnetic field $H_{l}$ acting at this site. For $J^{\prime}=J$ and $H_{l}=H_{d c}$, the Hamiltonian describes just the semi-infinite chain. Furthemore, let us denote $g^{z} \mu_{\mathrm{B}} H_{d c} \equiv H$ and $g^{z} \mu_{\mathrm{B}} H_{l} \equiv H^{\prime}$. It turns out that the unitary transformation used above can be applied to the spin chain with the impurity. Notice that the following results do not depend on the position of the impurity [11].

Then, using the Jordan-Wigner transformation [12]

$$
\begin{aligned}
& S_{n}^{+}=\prod_{m<n}\left(1-2 b_{m}^{\dagger} b_{m}\right) b_{n}, \\
& S_{n}^{-}=b_{n}^{\dagger} \prod_{m<n}\left(1-2 b_{m}^{\dagger} b_{m}\right),
\end{aligned}
$$

where $b_{n}\left(b_{n}^{\dagger}\right)$ are destruction (creation) operators of a fermion in the $n$-th site of the chain, we can rewrite the Hamiltonian $\mathcal{H}_{0}$ as

$$
\mathcal{H}_{0}=\sum_{n=1}^{L}\left[H b_{n}^{\dagger} b_{n}-(J / 2)\left(b_{n}^{\dagger} b_{n+1}+\text { H.c. }\right)\right]+H^{\prime} b_{0}^{\dagger} b_{0}-\left(J^{\prime} / 2\right)\left(b_{0}^{\dagger} b_{1}+b_{!}^{\dagger} b_{0}\right)+C,
$$

where $C$ is the operator-independent part of the Hamiltonian, and $L$ is the length of the chain (for the semiinfinite chain we use $L \rightarrow \infty$ ). For the homogeneous interaction $J_{n}=J$, the Hamiltonian can be easily diagonalized with the help of the Fourier transform and we obtain $\mathcal{H}_{0}=\sum_{k^{\prime}} \epsilon_{k^{\prime}} b_{k^{\prime}}^{\dagger} b_{k^{\prime}}$. For the infinite chain, it is possible to obtain the characteristics of all states of the Hamiltonian $\mathcal{H}_{0}$, see, e.g., [11] using the unitary transformation $b_{n}=(2 / L) \sum_{\lambda} u_{n, \lambda} a_{\lambda}$, where $\lambda$ denotes either band eigenstates or possible localized levels. There can exist band (extended) states and localized states. The band states (with their wave functions renormalized due to the coupled impurity) have the energy $\epsilon_{k}=H-J \cos k$, where $-\pi \leqslant k \leqslant \pi$. The localized states appear if the following conditions hold. Let us define $\Delta=\left(H^{\prime}-H\right) / J$ and $f=J^{\prime} / J$. Then, the local level with the energy $\epsilon_{1}$ and the local level with the energy $\epsilon_{2}$ are split from the upper/lower edge of the band if $f^{2}>2-2 \Delta$, or $f^{2}>2+2 \Delta$, respectively. The energies are [11] $\epsilon_{1,2}=H-J\left(f^{2}-1\right)^{-1}\left[\Delta\left(2-f^{2}\right) \pm f^{2} \sqrt{\Delta^{2}+f^{2}-1}\right]$. The localization lengths for those local levels are $\xi_{1,2}=\ln \left[\left(\Delta \pm \sqrt{\Delta^{2}+f^{2}-1}\right) /\left(f^{2}-1\right)\right.$. For $f=1$, we have only one level with $\epsilon=H+J\left(1+\Delta^{2}\right) / 2 \Delta$ with the localization length $\xi=-\ln (-2 \Delta)$.

\section{Dynamical susceptibility and the absorption of the ac magnetic field: homogeneous case}

In what follows we exactly consider the resonance terms explicitly dependent on time, which produce a nonzero contribution to the linear response. The remaining terms with an explicit time dependence can be omitted due to the smallness of the magnitude of the ac magnetic field $h_{1}, h_{1} \ll \hbar(\omega-m \Omega) \sim \epsilon_{k}, \epsilon_{1,2}$, standard for the magnetic resonance problem. Contribution from the non-resonance terms can be, in principle, calculated in the framework of the perturbation theory.

Let us first consider the homogeneous chain. For $J>0$, we can obtain for the ground state imaginary part of the dynamical susceptibility

$$
\bar{\chi}^{\prime \prime}(k, \omega)=\sum_{m=0, \pm 1, \pm 2, \ldots} \mathrm{e}^{-\mathrm{i} k m} \int_{-\infty}^{\infty} \mathrm{d} t \mathrm{e}^{\mathrm{i} \omega t}\left\langle b_{n}^{\dagger} b_{n+m}\right\rangle / 2 \hbar .
$$




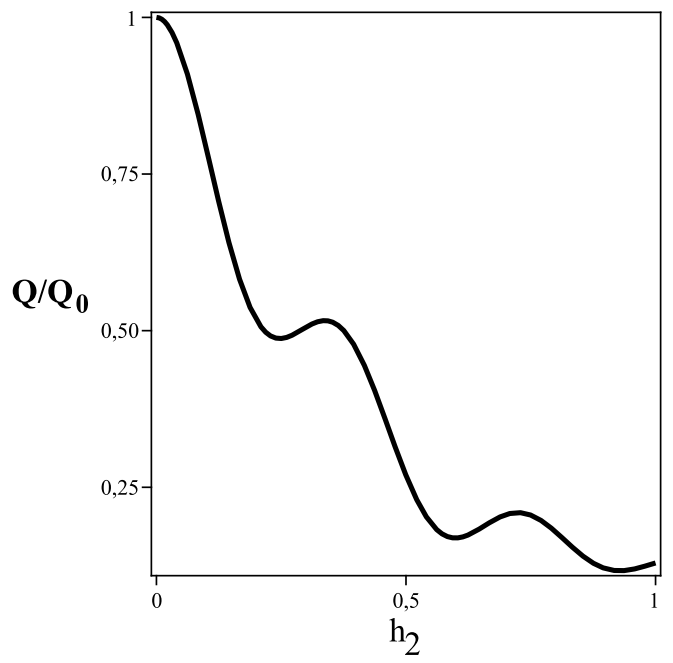

Figure 1. The relative strength of the combined resonance absorption $Q / Q_{0}$ by a homogeneous spin chain of the bi-chromatic ac field with respect to the one of the only circularly polarized ac field as a function of the magnitude of the linearly polarized ac field $h_{2}$. The values of the parameters $\hbar=1, \omega=10$ and $\Omega=0.1$ (arbitrary units), and $m=0,1,2,3$ were used.

For $J<0$, the additional factor $(-1)^{m}$ appears. For the absorption of the ac magnetic field, homogeneous along the chain, we can use $k=0$ (for both cases). Notice that such simple results can be obtained for the ground state only. For nonzero temperatures, the calculation of the correlation functions is much more complicated. However, for $H>|J|$ we can use the results [13], see also [14-16] for the circularly polarized field, see also [17] for the bi-chromatic field, using which it is possible to show that $\chi^{\prime \prime}(k, \omega-m \Omega) \sim(\pi / 2 \hbar) \delta(\hbar(\omega-m \Omega)-|H-| J \mid \cos k)$ for a homogeneous chain. The absorption of the ac magnetic field $Q$ is defined as $Q=\left\langle\partial \mathcal{H}_{t} / \partial t\right\rangle_{t a v}$, where brackets denote the ensemble averaging with the density matrix, and the index tav denotes the time averaging $A_{t a v}=\lim _{\tau \rightarrow \infty} \tau^{-1} \int_{0}^{\tau} A(t) \mathrm{d} t$. Absorption of the bi-chromatic ac magnetic field in the linear response regime can be written as

$$
Q=\frac{\left(h_{1}\right)^{2}}{2} \sum_{m} J_{m}^{2}\left(h_{2} / \hbar \Omega\right)(\omega-m \Omega) \chi(k=0, \omega-m \Omega) .
$$

The number $m$ determines which frequencies $\omega$ and $\Omega$ satisfy the resonance condition. Obviously, for $m=0$ and $h_{2}=0$, we obtain the previously known result, see, e.g., [14-16].

Figure 1 shows the relative strength of the absorption $Q$ by the considered homogeneous spin chain of the bi-chromatic ac magnetic field divided by the absorption $Q_{0}$ by the same chain of the only circularly polarized ac magnetic field as a function of the magnitude of the linearly polarized field $h_{2}$. Here, we used (arbitrary) units $\omega=10$ and $\Omega=0.1$ with resonances existing for $m=0,1,2,3$. We see that the linear polarized component of the ac field produces additional resonances with $m= \pm 1, \pm 2, \ldots$, with respect to the case of the only circular polarized ac field, which corresponds to the case $m=0$ and $h_{2}=0$. The absolute value of the Bessel function is the decaying oscillating function of its argument, hence the absorption also shows modulations. Even for the case $m=0, h_{2} \neq 0$, the linear polarized component produces modulations of the absorption. Contributions from the resonances with larger values of $m$ become (as a rule) essential for larger values of $h_{2} / \hbar \Omega$.

\section{Absorption by the impurity}

The problem of the response of the impurity to the bi-chromatic field is reduced to the problem of the response to the circularly polarized ac magnetic field, studied in [18], see also [19], with the necessary renormalization of the parameters. Here, it is possible to calculate the linear response for any temperature, not only for the ground state, as for the homogeneous chain. 
The absorption by the impurity can be written as $Q=Q_{m}^{(b)}+Q_{m}^{(l)}$, where the absorption due to the band states of the spin chain is

$$
\begin{aligned}
Q_{m}^{(b)} & =\frac{h_{1}^{2}(\omega-m \Omega) J_{m}^{2}\left(h_{2} / \hbar \Omega\right)}{2 J} \tanh \left[\frac{\hbar(\omega-m \Omega)}{2 k_{\mathrm{B}} T}\right] \\
& \times \frac{2 f^{2} \sqrt{1-x^{2}}}{4(\Delta+x)^{2}-4 f^{2} x(\Delta+x)+f^{4}} \Theta(1-|x|),
\end{aligned}
$$

where $x=H-\hbar \omega+m \hbar \Omega / 2 J, \Theta$ is the Heaviside step function, $T$ is the temperature, and $k_{\mathrm{B}}$ is Boltzmann's constant. On the other hand, the absorption caused by the local levels is

$$
\begin{aligned}
Q_{m}^{(l)} & =\frac{h_{1}^{2}(\omega-m \Omega) \pi J_{m}^{2}\left(h_{2} / \hbar \Omega\right)}{2} \tanh \left[\frac{\hbar(\omega-m \Omega)}{2 k_{\mathrm{B}} T}\right] \\
& \times\left[\delta ( \epsilon _ { 1 } - \hbar \omega + m \hbar \Omega ) \Theta ( f ^ { 2 } - 2 + 2 \Delta ) \left(-\frac{1}{f^{2}-1}\right.\right. \\
& \left.+\frac{f^{2}}{2\left(\Delta^{2}+f^{2}-1+\Delta \sqrt{\left.\Delta^{2}+f^{2}-1\right)}\right.}\right) \\
& +\delta\left(\epsilon_{2}-\hbar \omega+m \hbar \Omega\right) \Theta\left(f^{2}-2-2 \Delta\right)\left(-\frac{1}{f^{2}-1}\right. \\
& \left.\left.+\frac{f^{2}}{2\left(\Delta^{2}+f^{2}-1-\Delta \sqrt{\left.\Delta^{2}+f^{2}-1\right)}\right.}\right)\right]
\end{aligned}
$$

where $\delta(x)$ is the Dirac delta-function. One can see that for $J^{\prime}=0$ (i.e. $f=0$ ) the absorption from the second local level is zero, while the absorption from the first one becomes $Q_{m}^{(l)} \sim \delta\left[H^{\prime}-\hbar(\omega+m \Omega)\right]$, i.e., it is the absorption of a single impurity. The absorption caused by the local levels, and by the extended states decays with the growth of the temperature.

Again, the linear polarized component of the ac field produces additional resonances for the absorption caused by the local levels and extended states with $m= \pm 1, \pm 2, \ldots$, with respect to the case of the only circular polarized ac field, which corresponds to the case $m=0$ and $h_{2}=0$. Contributions from the resonances with larger values of $m$ become (as a rule) essential for larger values of $h_{2} / \hbar \Omega$.

Figure 2 shows the absorption by the impurity in the spin chain as a function of the frequency of the circularly polarized ac magnetic field $\omega$. We used parameters $\hbar=1, H=4, H^{\prime}=7, J=0.18, J^{\prime}=9$, $h_{2}=1, \Omega=0.5, k_{\mathrm{B}} T=0.1$, and we used the half-linewidth for the resonances, related to the local levels, as $\gamma=0.01$. For that set of parameters, only one local level exists. For convenience, we used $1 / 5$ of the values for the main resonance $m=0$.

Figure 3 shows the combined resonance absorption for $m=-1$ as a function of the frequency of the circularly polarized field $\omega$ and the parameter of the linearly polarized field $h_{2} / \hbar \Omega$. We used the set of parameters $\hbar=1, H=2, H^{\prime}=0.1, J=4, J^{\prime}=2$, and $\Omega=0.5$ at the temperature $k_{\mathrm{B}} T=0.1$. For this set of parameters there are no local levels, and the absorption by the impurity exists only due to the extended (band) states. We see decaying oscillations of the absorption as a function of the magnitude of the linearly polarized field $h_{2}$.

\section{Summary}

In summary, we have calculated the linear response of the spin-1/2 chain with the impurity to the bi-chromatic ac magnetic field. We have shown that the presence of the linearly polarized field produced additional resonance absorption at the combined frequencies of the circularly polarized and linear polarized ac field. The strength of the absorption oscillates with the amplitude and the frequency of the linearly polarized ac magnetic field. Additional resonance absorption is weaker than the main absorption at the frequency of the circularly polarized field. For combined resonances, the relative weights of the short-wavelength and long-wavelength wings of the absorption are different from the 


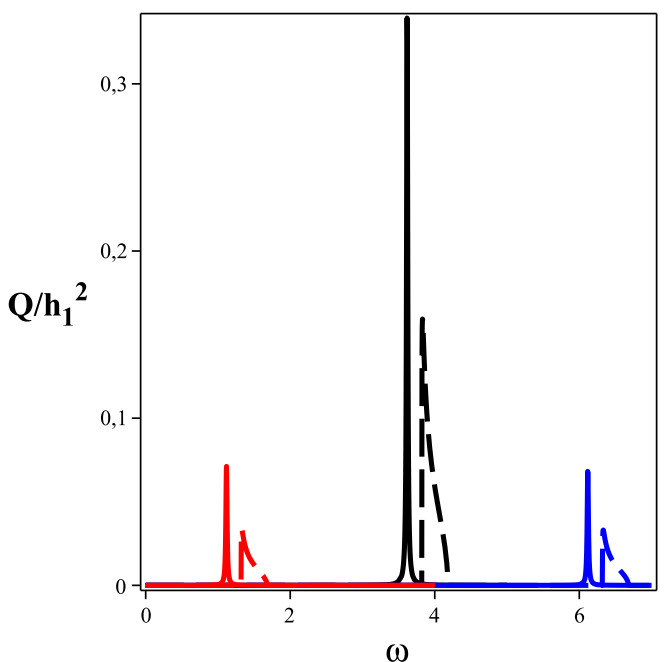

Figure 2. (Color online) The combined resonance absorption (normalized by the energy of the ac field $h_{1}^{2}$ ) $Q / h_{1}^{2}$ by the spin chain with the impurity as a function of the frequency of the circularly polarized field $\omega$. Solid lines describe the contribution from the local level. Dashed lines describe the contribution of the extended states. Black lines: the main resonance $m=0$; red lines: the combined resonance $m=-1$; blue lines: the combined resonance $m=1$. The parameters used are $\hbar=1, H=4, H^{\prime}=7, J=0.18$, $J^{\prime}=9, h_{2}=1, \Omega=0.5, k_{\mathrm{B}} T=0.1$, and we used the half-linewidth for the resonances related to the local levels, as $\gamma=0.01$.

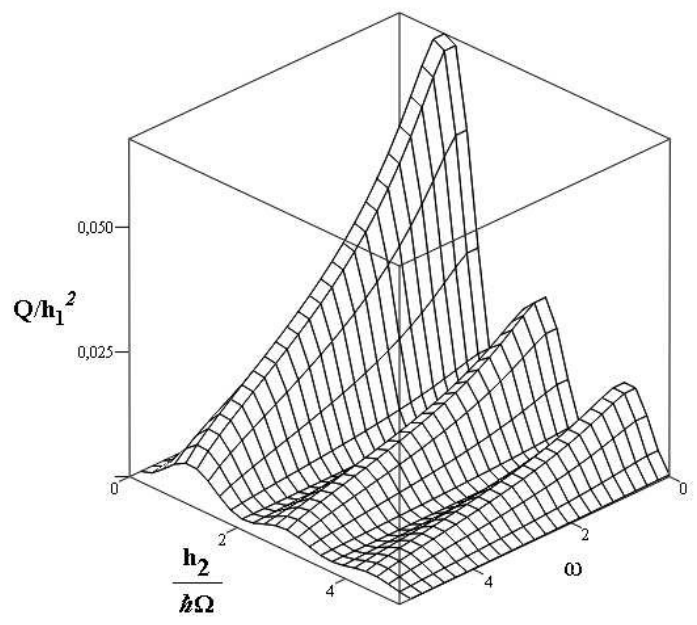

Figure 3. The combined resonance $m=-1$ absorption (normalized by the energy of the ac field $h_{1}^{2}$ ) $Q / h_{1}^{2}$ by the spin chain with the impurity as a function of the frequency of the circularly polarized field $\omega$ and the parameter of the linearly polarized ac field $h_{2} / \hbar \Omega$.

ones of the main resonance (only circularly polarized field). We have also shown that the absorption by the impurity can be divided into two parts: the one by the possible localized states, and the one by the extended states. Both contributions also manifest combined resonances due to the bi-chromatic ac field.

\section{References}

1. Lee P.A., Science, 2008, 321, 1306-1307, doi $10.1126 /$ science.1163196

2. Savary L., Balents L., Rep. Progr. Phys., 2017, 80, 016502, doi $10.1088 / 0034-4885 / 80 / 1 / 016502$

3. Zvyagin A.A. Quantum Theory of One-Dimensional Spin Systems, Cambridge Scientific Publishers, Cambridge 2010 . 
4. Mermin N.D., Wagner H., Phys. Rev. Lett., 1966, 17, 1133-1136, doi:10.1103/PhysRevLett.17.1133

5. Purcell E.L., Phys. Rev., 1946, 69, 681, doi 10.1103/PhysRev.69.37.

6. Drexhage K.H. In: Progress in Optics, 12, Wolf E. (Ed.), Elsevier, New York, 1974, 163-232.

7. Chance R.R., Prock A., Silbey R., In: Advances in Chemical Physics, Vol. 37, Prigogine I., Rice S.A. (Eds.), John Wiley \& Sons, Inc., New York, 1978, 1-65, doi 10.1002/9780470142561.ch1.

8. Kleppner D., Phys. Rev. Lett., 1981, 47, 233-236, doi 10.1103/PhysRevLett.47.233

9. Lodahl P., van Driel A.F., Nikolaev I.S., Irman A., Overgaag K., Vanmaekelbergh D.A.M., Vos W.L., Nature, 2004, 430, 654-657, doi 10.1038 /nature02772.

10. Zvyagin A.A., Phys. Rev. B, 2020, 101, 174408, doi $10.1103 /$ PhysRevB.101.174408

11. Zvyagin A.A., Finite Size Effects in Correlated Electron Models: Exact Results, Imperial College Press, London, 2005.

12. Jordan P., Wigner E., Z. Phys., 1928, 47, 631651, doi:10.1007/BF01331938

13. Cruz H.B., Goncalves L.L., J. Phys. C: Solid State Phys., 1981, 14, 2785-2792, doi $10.1088 / 0022-3719 / 14 / 20 / 016$

14. Derzhko O., Krokhmalskii T., Phys. Rev. B, 1997, 56, 11659-11665, doi 10.1103/PhysRevB.56.11659

15. Derzhko O., Krokhmalskii T., Stolze J., J. Phys. A: Math. Gen., 2000, 33, 3063-3080, doi $10.1088 / 0305-4470 / 33 / 16 / 301$

16. Verkholyak T., Derzhko O., Krokhmalskii T., Stolze J., Phys. Rev. B, 2007, 76, 144418, doi $10.1103 /$ PhysRevB.76.144418.

17. Zvyagin A.A., Ropakova I.Yu., Fiz. Nizk Temp., 2020, 46, 805-811 [Low Temp. Phys., 2020, 46, N7, 677, doi $10.1063 / 10.0001363$ ].

18. Kleiner V.Z., Tsukernik V.M., Fiz. Met. Metalloved., 1975, 39, 947-951 (in Russian).

19. Zvyagin A.A., Phys. Rev. B, 2010, 82, 104403, doi $10.1103 /$ PhysRevB.82.104403.

\title{
Вплив біхроматичного змінного магнітного поля на квантовий спіновий ланцюжок з домішкою
}

\author{
A.A. Звягін \\ ${ }^{1}$ Інститут Макса Планка фізики комплексних систем, вул. Ньотнитцер 38, 01187 Дрезден, Німеччина \\ 2 Фізико-технічний інститут низьких температур ім. Б.І. Вєркіна НАН України, пр. Науки 47, \\ 61103 Харків, Україна
}

Розраховано лінійний відгук квантового спінового ланцюжка з домішкою на біхроматичне змінне магнітне поле. Лінійно поляризована компонента змінного поля призводить до додаткового резонансного поглинання відносно компоненти викликаної циркулярно поляризованим полем. Сила поглинання осцилює з амплітудою та частотою лінійно поляризованого магнітного поля. Додаткове резонансне поглинання виявляється слабшим ніж основне поглинання на частоті циркулярно поляризованого поля. Поглинання домішкою можна побачити у поглинанні на локалізованих рівнях та делокалізованих станах.

Ключові слова: біхроматичне змінне магнітне поле, квантовий спіновий ланцюжок, домішка 


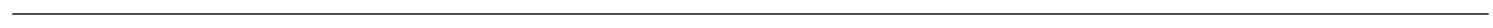

